\title{
A ELISÃO EM CONTEXTOS DE VOGAIS ADJA- CENTES: IMPLICAÇÕES PARA A TEORIA DA OTIMALIDADE
}

ELISION IN ADJACENT VOWEL CONTEXTS: IMPLICATIONS FOR OPTIMALITY THEORY

\section{Gisela Collischonn ${ }^{1}$}

Resumo: Este trabalho discute alguns fatores que condicionam a elisão de /a/ em fronteira de palavra e sua interpretação em termos de teoria fonológica. Focalizamos o comportamento de sequências em que a vogal baixa é precedida por uma vogal anterior alta, como no exemplo dia inteiro. A argumentação baseia-se na perspectiva de que o processo de elisão é um efeito de restrições em conflito. Em sequências do tipo /...ia\#i.../, a elisão resulta uma sequência [...ii...], que é uma estrutura marcada e, no português brasileiro, é desfeita em outros contextos ou leva a efeitos de bloqueio. Por outro lado, a elisão é uma das escolhas preferidas para esses contextos (d[ii]nteiro), conforme mostram dados de Ludwig Gayer (2008). As consequências para uma abordagem não-derivacional da fonologia são analisadas.

Palavras-chave: sândi vocálico, elisão, Teoria da Otimidade, restrições.

Abstract: This chapter considers some factors that condition elision of /a/ at word boundaries and their interpretation in terms of phonological theory. We focus on the behavior of sequences where the vowel to be deleted follows a high vowel, as in the example dia inteiro "whole day". The discussion is based on the perspective that the elision process is an effect of restrictions in conflict. In sequences of the /...ia \#i.../ type, elision of the vowel /a/ yields a sequence [... ii...], which is a marked structure that is undone in other contexts, in Brazilian Portuguese, or has a blocking effect on phonological processes. However, elision is one of the preferred choices in these contexts (d[ii]nteiro), as show data from Ludwig Gayer (2008). The consequences of these findings for a non derivational approach to phonology are analyzed.

Keywords: vowel sandhi; elision; Optimality Theory; constraints.

1 Professora do Instituto de Letras da UFRGS, pesquisadora do CNPq. 


\section{Introdução}

Neste trabalho, analisamos o fenômeno de sândi chamado de elisão, como observado no exemplo roupz inteirinha. A análise busca entender os fatores que condicionam a elisão quando a sequência alvo contém mais de duas vogais e discute sua interpretação em termos de teoria fonológica. São da amostra de São Borja-RS do Banco VARSUL os dados que consideramos aqui.

A amostra interessa-nos porque foi a base de uma pesquisa sobre os processos de sândi vocálico, em pesquisa realizada nos moldes da teoria da variação por Ludwig-Gayer (2008). Os resultados desse levantamento confirmam, em linhas gerais, os de outros, como Bisol (2002 a e b) e Tenani (2002). O que se observa é que, no português brasileiro, mesmo que as taxas de elisão possam se alterar de uma amostra para a outra, isto é, o valor de "input" pode variar, os fatores condicionadores permanecem razoavelmente constantes. ${ }^{2}$ Abaixo, apresentamos alguns trabalhos realizados sobre o fenômeno da elisão e sua caracterização em termos de amostra, número de dados e de informantes e principais resultados.

\begin{tabular}{|c|c|c|c|c|c|c|}
\hline Publicação & $\begin{array}{c}\text { Origem dos } \\
\text { dados }\end{array}$ & $\begin{array}{c}\mathrm{N}^{\circ} \text { de } \\
\text { sujeitos }\end{array}$ & Localidade & $\begin{array}{c}\text { Número de } \\
\text { dados }\end{array}$ & $\begin{array}{c}\text { Porcentagem de } \\
\text { aplicação }\end{array}$ & Peso relativo \\
\hline Bisol (2002a) & VARSUL & 12 & Porto Alegre & 1588 & $32 \%$ & 0,21 \\
\hline Bisol (2002b) & NURC & & & 605 & $11 \%$ & \\
\hline Tenani (2002) & $\begin{array}{c}\text { Coletados } \\
\text { pela autora }\end{array}$ & 3 & São Paulo & 96 & $74 \%$ & \\
\hline $\begin{array}{c}\text { Ludwig-Gayer } \\
\text { (2008) }\end{array}$ & VARSUL & 8 & São Borja-RS & 784 & $55 \%$ & 0,56 \\
\hline Vianna (2009) & VARSUL & 16 & Florianópolis & 835 & $33 \%$ & 0,30 \\
\hline Peixoto (2011) & VARSUL & 10 & Lages-SC & 1000 & $46 \%$ & 0,42 \\
\hline
\end{tabular}

Quadro 1: Resultados de pesquisas sobre elisão, com informação da base de dados e do número de dados analisados

Os fatores condicionadores que se observaram relevantes nos trabalhos analisados são: acento, domínio prosódico e qualidade da vogal seguinte.

$2 \mathrm{O}$ valor de input em uma análise pelo programa VARBRUL/GoldVARB refere-se à média corrigida, i.e., à medida global da taxa de aplicação de uma determinada regra. O input pode ser entendido como "uma freqüência média de ocorrência do valor de aplicação da variável dependente" (Paolillo 2002: 79). Na pesquisa variacionista, podemos considerar que os resultados se assemelham entre amostras, quando os fatores condicionadores tiverem um papel constante, ainda que o valor de input seja diferente. Veja-se a respeito Tagliamonte (p. 242 ss.). 
Abaixo, resumimos as principais conclusões: ${ }^{3}$

(i) o acento frasal (nuclear) em $\mathrm{V}_{2}$ favorece manutenção de hiato ((zon $\underline{\boldsymbol{a}}$ urbana) vs para outro lugar)) e

(ii) a taxa de elisão está inversamente correlacionada à possibilidade de encontro acentual;

(iii) a elisão se aplica preferentemente internamente à frase fonológica (guerra econômica) do que entre frases ( a Caixa incorporou);

(iv) a elisão se aplica mais em contextos em que a vogal baixa precede vogais posteriores arredondadas na palavra seguinte, $a \#\{0, u\}$, do que em contextos a\#\{e,i\}, o que, em nosso entendimento, se explica a partir de uma preferência maior da língua por ditongos decrescente do tipo [aj] do que do tipo [aw]; em outras palavras, não é uma característica fonética da vogal posterior /o,u/ que favorece o apagamento da vogal /a/ precedente, mas a probabilidade maior de uma sequência /a+ vogal anterior/ de realizar-se como ditongo decrescente que favorece menos a elisão da vogal baixa nestes contextos. ${ }^{4}$

A análise de Ludwig-Gayer (2008) mostrou que a elisão não é influenciada por variáveis sociais, tais como idade, sexo ou nível de escolaridade. Não foi encontrada diferença significativa entre os informantes, indicando que a aplicação do processo é altamente uniforme na comunidade de fala considerada e que este não funciona como marcador social. Resultados semelhantes foram observados por Bisol (2002a) e por Vianna (2009) ${ }^{5}$. Portanto, trata-se de um fenômeno não-categórico, de aplicação opcional, mas não se trata de mudança em curso.

Em relação a estes resultados, o problema principal deste artigo refere-se ao tratamento de sequências em que $\mathrm{V}_{1}$ é precedida por uma vogal anterior alta, como no exemplo "dia inteiro". Tais dados são interessantes porque a aplicação da elisão em uma sequência /...ia\#i.../ leva a uma seqüência de vogais idênticas, uma configuração que é pouco tolerada no português brasileiro. Atestam isso dois tipos de evidência: por um lado, processos de

3 Tenani (2002) não analisou o papel da vogal seguinte, se anterior ou posterior, na taxa de aplicação.

4 Evidências diacrônicas apontam para isso. Conforme Quednau (2005), houve criação de ditongos [aj] no latim vulgar, ao passo que os ditongos [aw] foram monotongados ou passaram a [ow]. O português brasileiro, entretanto, difere do europeu, no qual a formação de ditongo decrescente entre palavras somente ocorre se a segunda vogal for arredondada (Vigário 2003, p. 101).

5 É preciso reconhecer que, na análise de Bisol (2002a), o fator "escolaridade" foi selecionado como estatisticamente relevante. $\mathrm{Na}$ análise de Vianna (2009), nenhum fator extralinguístico foi selecionado. 
degeminação em fronteira de palavra; e, por outro, e bloqueios de formação morfológica, por exemplo, com -inho ( ${ }^{\star}$ friinho, ${ }^{\star}$ riinho, ${ }^{\star}$ arrepiinho). Esperar-se-ia, portanto, que as mesmas restrições atuantes nos processos de degeminação e de bloqueio morfológico atuassem também no sentido de evitar a aplicação da elisão sempre que o resultado fosse uma sequência de vogais idênticas.

Contrariando as expectativas, um levantamento nos dados analisados por Ludwig-Gayer (2008) mostra que a elisão é uma das escolhas preferidas para esses contextos (d[ii]nteiro). As consequências desta descoberta para uma abordagem não-derivacional da fonologia são analisadas.

O artigo tem a seguinte organização: na próxima seção, apresenta-se uma breve análise da elisão e das restrições que dão conta do processo em uma análise otimalista; na seção seguinte, a análise das sequências ia\#[i $]^{6}$ é aprofundada; o problema do paradoxo de ranking é destacado na quarta seção e discute-se até que ponto uma abordagem pelo Serialismo Harmônico poderia tratar as seqüências ia\#[i]; mostramos que a proposta não dá conta de explicar os dados. A seção final apresenta as principais conclusões e projeções.

\section{Análise da elisão e das restrições em jogo}

Nesta seção, apresentamos as restrições que permitem analisar o processo de elisão na perspectiva da Teoria da Otimalidade, bem como os argumentos que sustentam o ranking dessas restrições. Ao final da seção, voltamo-nos para a análise das restrições envolvidas em processos de simplificação de sequências de vogais idênticas. O objetivo é de apresentar os elementos que, juntamente com a análise das sequências de três vogais /ia\#i/, formam as bases para o problema de paradoxo de ranking, discutido na seção 4 .

Como se pode observar nos exemplos abaixo, no processo de elisão, a restrição de fidelidade Max V é violada para satisfazer a restrição que milita contra a existência de hiato. Neste trabalho, consideraremos que tal restrição é Onset. ${ }^{7}$

6 Representamos V2 entre colchetes para destacar que, subjacentemente, a vogal pode ser a vogal /e/, realizada foneticamente como [i].

7 Em outro trabalho (COLLISCHONN, 2012), consideramos que a restrição responsável pela elisão deva estar relacionada ao acento, para explicar a relação de precedência que se estabelece entre acento e elisão. Como a questão relativa à restrição não é o foco deste trabalho, ficaremos, por ora, com a restrição Onset. 
Exemplos;
a. er[a i]rmã
$/ \mathrm{a}_{1} \# \mathrm{i}_{2} / \rightarrow\left[\mathrm{i}_{2}\right]$
d. zon[a u]rbana
$/ \mathrm{a}_{1} \# \mathrm{u}_{2} / \rightarrow\left[\mathrm{u}_{2}\right]$

Em geral, numa sequência $\mathrm{V} \# \mathrm{~V}$, vogais com acento primário tendem a ser preservadas. Por isso, um /a/ tônico seguido de outra vogal nunca é elidido (está imenso). A questão sobre o papel que o acento exerce como bloqueador da elisão foi tratada em outros trabalhos, por exemplo, em Bisol (2003), como resultante da atribuição prévia de acento, numa proposta de avaliação em dois níveis. Em Collischonn (2012), mostramos que a questão do papel do acento, para uma abordagem baseada em avaliação em paralelo, como a Teoria da Otimalidade, é complexa, pois o acento nunca parece ser determinado pela elisão ou por outro processo de sândi, configurando uma situação que McCarthy (2008) caracteriza como processo condicionado pelo acento.

Uma proposta interessante - porque relaciona o apagamento ao acento - é a de Wheeler (2005) para o catalão, que se baseia em duas restrições de marcação: Onset e ${ }^{\star}$ Lapso (atribua uma marca de violação para cada par de sílabas não acentuadas adjacentes). No entanto, ela parece não ser suficientemente geral, pois há casos em que o apagamento não repara a violação à restrição *Lapso (como em a, abaixo) e, por outro, há casos em que o apagamento ocorre mesmo não havendo lapso (como em b):

\begin{tabular}{|c|l|}
\hline $\begin{array}{c}\text { a.minh[a o]pinião } \\
\text { ' } \sigma \sigma \sigma \sigma^{\prime} \sigma\end{array}$ & $/ \mathrm{a}_{1} \# \mathrm{o}_{2} / \rightarrow\left[\mathrm{o}_{2}\right]$ \\
\hline $\begin{array}{c}\text { b. agor[a e]u } \\
\sigma^{\prime} \sigma \sigma^{\prime} \sigma\end{array}$ & $/ \mathrm{a}_{1} \# \mathrm{e}_{2} / \rightarrow\left[\mathrm{e}_{2}\right]$ \\
\hline
\end{tabular}

Portanto, em nosso entendimento, ${ }^{\star}$ Lapso não parece ser a melhor alternativa para Onset.

Neste momento, a questão da precedência do acento, embora relevante, não é crucial para a análise que vamos empreender e, por isso, iremos considerar que o acento esteja atribuído no input. Por essa razão, adotamos a análise com a restrição de fidelidade posicional, $\mathbf{M a x V}_{\text {acent }}$ - atribua uma marca de violação a cada vogal acentuada que não tenha correspondente no output (correspondência I-O, input-output, ou B-O, base-output). A generalização de que vogais com acento primário tendem a ser preservadas indica, portanto, para o seguinte: $\mathrm{MaxV}_{\text {acent. }}$ domina Onset

No tableau a seguir, considere-se que as formas representadas são er[a i] rmã, jacarand[á i]menso. Por questão de espaço, restringimos a represen- 
tação dessas formas apenas ao contexto relevante para a elisão. Neste e em diversos tableaux desta seção e das seções seguintes, será usado o formato do tableau comparativo (PRINCE, 2002, MCCARTHY, 2008). Neste tipo de tableau, diversos inputs e seus respectivos candidatos a output são analisados simultaneamente. A razão por que o tableau é chamado de comparativo é porque cada candidato a output é comparado ao pretenso ganhador. Em relação ao tableau standard da OT - chamado de tableau de violações -, o tableau comparativo é superior para a identificação de argumentos de ranking, principal objetivo das apresentações neste artigo. Neste tableau não há asteriscos, mas símbolos que identificam, para cada restrição, se esta escolhe o ganhador (W) ou o perdedor (L). ${ }^{8}$ Nos pares de candidatos, é sempre o da esquerda do símbolo $\prec$ que é o ganhador e o da direita o perdedor. O símbolo pode ser traduzido como "é mais harmônico que".

\begin{tabular}{|c|c|c|c|c|}
\hline Inputs & Candidatos & $\operatorname{MaxV}_{\text {acent }}$ & Onset & Max V \\
\hline a. /a\#i/ & {$[\mathrm{i}] \prec[\mathrm{a} . \mathrm{i}]$} & & $\mathrm{W}$ & $\mathrm{L}$ \\
\hline b. /á\#i/ & {$[\mathrm{a} . \mathrm{i}] \prec[\mathrm{i}]$} & $\mathrm{W}$ & $\mathrm{L}$ & $\mathrm{W}$ \\
\hline
\end{tabular}

Tableau 1: Tableau comparativo com dois inputs e dois candidatos por input

No tableau acima, o candidato [i], com apagamento de $V_{1}$, ganha quando $V_{1}$ não possui acento, como no input a., mas perde para o candidato sem apagamento quando $\mathrm{V}_{1}$ for acentuada, como no input b. Uma solução imaginável para satisfazer Onset neste caso seria apagar $V_{2}$ em vez de $V_{1}$. No entanto, há uma restrição MaxWI que milita contra este apagamento, definida como segue: MaxWI - atribua uma marca de violação a cada vogal que estiver no início de palavra no input e que não tiver correspondente no output (conforme CASALI, 1997).

Uma vez que essa restrição entra em jogo impedindo que Onset seja satisfeito, tem-se o seguinte ranking: $\mathrm{MaxV}_{\text {acent }}$, Max WI $>>$ Onset $>>$ MaxV. O tableau a seguir mostra a seleção de candidatos diante desse ranking. ${ }^{9}$

\begin{tabular}{|l|l|l:l|l|l|}
\hline Inputs & Candidatos & $\mathrm{MaxV}_{\text {acent }}$ & Max WI & Onset & Max V \\
\hline$/$ a\#\#i/ & {$[\mathrm{i}] \prec[\mathrm{a} . \mathrm{i}]$} & & & $\mathrm{W}$ & $\mathrm{L}$ \\
\hline$/$ á\#i $/$ & {$[\mathrm{a} . \mathrm{i}] \prec[\mathrm{i}]$} & $\mathrm{W}$ & & $\mathrm{L}$ & $\mathrm{W}$ \\
\hline & {$[\mathrm{a} . \mathrm{i}] \prec[\mathrm{a}]$} & & $\mathrm{W}$ & $\mathrm{L}$ & $\mathrm{W}$ \\
\hline
\end{tabular}

Tableau 2: Tableau comparativo com dois inputs, com dois e três candidatos, respectivamente

8 As siglas são emprestadas do inglês e estão, respectivamente, por winner e looser.

9 Neste tableau, são apresentados dois pares de candidatos para o input b. 
Quando, por sua vez, a segunda vogal for tônica (contra Isso), a elisão também é menos frequente, especialmente se nesta segunda vogal também incidir o acento nuclear (numa URna). Bisol (2003) explica esse tipo de condicionamento com uma restrição de alinhamento ALIGN-L, que diz que a borda esquerda do pé de acento frasal deve coincidir com a borda esquerda de uma palavra lexical. ${ }^{10}$

Voltando agora nossa atenção às seqüências ia\#[i] ou ua\#[u], caracterizaremos esquematicamente as vogais como $\mathrm{V}_{0} \mathrm{~V}_{1} \# \mathrm{~V}_{2}$. A supressão de $\mathrm{V}_{1}$ pode criar sequências $\left[\mathrm{i}_{0} \mathrm{i}_{2}\right]$ ou $\left[\mathrm{u}_{0} \mathrm{u}_{2}\right]$, que são marcadas em português. As razões por que as consideramos marcadas são apresentadas a seguir.

Em primeiro lugar, há evidências tanto na morfologia derivacional quanto na flexional de que sequências de vogais idênticas que são criadas através da concatenação de morfemas são desfeitas de alguma forma ou bloqueiam determinado processo morfológico. Na morfologia derivacional, sequências desse tipo ocorrem esporadicamente (por exemplo, fri-issimo "frio + grau superlativo"), mas, na maioria das vezes, são evitadas. ${ }^{11}$ Por exemplo, folia e mania não admitem diminutivos com inh $V^{\star}$ foliinha, * maniinha, mas apenas com -zinhV - foliazinha e maniazinha. Formas como canoa e garoa, por seu lado, admitem diminutivos com -inhV - canoinha, garoinha. Isso mostra que o problema com folia e mania não está no fato de estes radicais terminarem em vogal, $\left[[\text { foli }]_{\operatorname{Rad}} a_{\mathrm{VT}}\right]_{\mathrm{Palavra}} \mathrm{e}[[\mathrm{mani}]$ $\left.{ }_{\text {Rad }} a_{\mathrm{VT}}\right]_{\text {Palavra }}$. Podemos, portanto, supor que a não formação de diminutivos * foliinha e *maniinha reside no fato de que sequências /ii/ são evitadas sempre que possível. ${ }^{12}$

Em segundo lugar, na flexão, estas sequências, quando surgem da concatenação de morfemas, também são evitadas com degeminação - por exemplo /part-+-i+ -is/ é realizado como [par't fis] - ou, às vezes, com inserção de glide / atu-+ - o / [a’tuwo].

10 Novamente, a abordagem supõe uma avaliação em etapas, com o acento sendo atribuído anteriormente à aplicação da elisão. Neste trabalho, não aprofundaremos esta discussão, mas cabe registrar novamente que uma abordagem plenamente paralela da Teoria da Otimalidade, acrescida da suposição de que o acento não está no input, enfrentaria complexidade adicional para dar conta desse aspecto.

11 Sobre as formas em -íssimo, o dicionário Houaiss registra com o hiato ií as seguintes formas: feiíssimo, fríssimo, maciíssimo (com variante macilíssimo), piíssimo, propriíssimo e seriíssimo (com variante seríssimo). Diversas gramáticas registram as variantes feíssimo, cheíssimo, sumaríssimo e dizem que o português atualmente prefere as formas com um só [i]. Consideramos que formas como sedentaríssimo, precaríssimo, solidaríssimo sejam perfeitamente possíveis.

12 Há que observar aqui que formas como o hipotético foliinha não podem ser resolvidas através de degeminação, porque o acento sobre a segunda vogal $i$ bloqueia o processo. Essa observação não invalida a constatação de que seqüências ii são evitadas na língua.

Organon, Porto Alegre, v. 28, n. 54, p. 257-278, jan./jun. 2013. 
$\mathrm{Na}$ fonologia frasal, sequências de vogais idênticas, como aqui\#importa e tu\#usavas tendem a ser resolvidas por degeminação. O processo atinge não somente vogais idênticas, mas também sequências de vogais com o mesmo ponto de articulação, exemplo sempre\#ignorava, feito\#uso. $\mathrm{Na}$ amostra da qual se originam os dados deste trabalho, a degeminação em fronteira de palavras foi observada por Ludwig-Gayer (2008, p. 64), que constatou que, em cerca de $76 \%$ das ocorrências analisadas, o processo se aplicou, índice que fica mais alto quando se consideram sequências de vogais não acentuadas (82\%).

Portanto, essas observações nos levam a supor que exista uma restrição do tipo ${ }^{*} \mathbf{V}_{\alpha} \mathbf{V}_{\alpha}$ (WHEELER, 2005), que penaliza hiatos entre vogais de mesma qualidade. Para que a restrição tenha efeito, ela deve estar ranqueada acima de Max V.

Portanto, os rankings parciais a que chegamos, até aqui são:

a) $\mathrm{MaxV}_{\text {acent, }}$ Max WI $>>$ Onset $>>\mathrm{MaxV}$

b) ${ }^{*} V_{a} V_{a}>>M a x V$.

\section{As sequências ia\#[i]}

Nesta seção, apresentamos os dados que são foco da análise, buscando orientar o olhar para a questão fundamental que será abordada na seção seguinte. Como se trata de dados com realização variável, procuramos caracterizar em linhas gerais as gramáticas que geram as diferentes variantes.

Para o levantamento que apresentamos aqui, buscamos, no conjunto de dados analisado por Luwig-Gayer (2008), todas as sequências em que $\mathrm{V}_{1}$ fosse precedida por uma vogal (ou semivogal) anterior alta e $\mathrm{V}_{2}$ também fosse uma vogal anterior alta (ou vogal com propensão a se realizar como alta). A seguir, apresentamos alguns dos exemplos recolhidos (o tachado duplo indica uma vogal que foi suprimida na realização observada):

\begin{tabular}{|c|c|c|c|}
\hline a. & gostaria imensamente & d. & podia ir \\
\hline b. & dia inteiro & & ia indo \\
\hline & iria explodir & e. & farmacia inteira \\
\hline c. & loteria e & & propria experiencia \\
\hline
\end{tabular}

Foram encontradas 24 ocorrências, das quais 17 apresentaram elisão da vogal (71\%). ${ }^{13}$ Isso mostra que a elisão não é evitada quando resulta

13 A análise foi de oitiva. Algumas

Organon, Porto Alegre, v. 28, n. 54, p. 257-278, jan./jun. 2013. 
em uma sequência de vogais idênticas. Além da elisão, há também a possibilidade de manutenção da vogal baixa e, finalmente, a possibilidade de apagamento de duas vogais, com o resultado de que uma sequência de três vogais fica reduzida a uma.

Alternativas de realização

\begin{tabular}{|c|c|c|c|}
\hline ...ia\#i... & $\rightarrow$..ia\#i... & a. $\ldots i_{0} a_{1} i_{2} \ldots$ & manutenção de [a] \\
\hline & $\rightarrow \ldots$...ia\#i... & b. ... $\mathrm{i}_{0} \mathrm{i}_{2} \ldots$ & elisão \\
\hline & $\rightarrow$..jat\#i... & c. .... $\mathrm{i}_{0} \ldots$ & elisão + degeminação \\
\hline
\end{tabular}

Quadro 2: Possibilidades de realização da sequência de input ...iał\#i...

No nosso entendimento, se as alternativas não forem puramente de caráter fonético, deve haver uma gramática que explique a realização $\mathbf{b}$ tanto quanto outra gramática que explique a realização c. É importante esclarecer que a nossa perspectiva sobre a variação é a da existência de múltiplas gramáticas para dar conta da opcionalidade de aplicação, cf. Kroch (1989) e Kiparsky (1993) (ver Anttila, 2007, e Battisti, 2010). Nesta perspectiva, o falante possui diferentes rankings, cada um deles dando conta de uma das formas alternativas, cada ranking sendo uma gramática. Então, teoricamente, temos de encontrar um conjunto de restrições que, sob diferentes rankin$g s$, consiga gerar as formas a, b e c. Com as restrições que temos, é possível obter rankings que gerem a e c. Portanto, o foco agora é a realização $\mathbf{b}$.

Como trabalhamos no quadro da OT, a explicação se baseia na hipótese de que qualquer gramática que gere a realização $\mathbf{b}$ precisa explicar como b é mais harmônica do que a ou c. Isto é, deve haver restrições que favoreçam a seleção de $\mathbf{b}$ sobre a ou c. A pergunta é: que tipo de gramática permitiria o resultado $\mathbf{b}$, tendo em vista o output marcado que ela produz? No nosso entendimento, essa gramática atribui papel crucial às restrições de fidelidade.

Antes de prosseguirmos, é importante considerarmos uma hipótese alternativa para a ocorrência de $\mathbf{b}$ (que será descartada): a de que a forma $\mathbf{c}$, que é a forma menos marcada, não se realizaria em alguns casos porque a degeminação subsequente à elisão seria bloqueada, por não encontrar contexto adequado de aplicação. Consideramos duas possibilidades: (a) casos em que o acento incide em $\mathrm{V}_{0}$, que poderiam ser explicados por algum tipo de bloqueio ao apagamento de sílaba acentuada e (b) casos em que a estrutura da sílaba de $\mathrm{V}_{2}$ contém uma coda nasal, nos quais o bloqueio poderia ser devido ao fato de não haver plena identidade entre as duas vogais a serem degeminadas. Abaixo mostramos que não há bloqueio de degeminação quando $V_{0}$ for acentuada, mas somente quando $V_{2}$ for acentuada. Também mostramos que não há bloqueio quando $\mathrm{V}_{2}$ for nasal. 


\begin{tabular}{|c|c|c|}
\hline $\begin{array}{l}\operatorname{gostari}_{0} \mathrm{a}_{1} \text { i mensamente } \\
\sigma \quad \sigma^{\prime} \sigma \sigma \quad \sigma^{\prime} \sigma \quad \sigma^{\prime} \sigma \quad \sigma\end{array}$ & ...ía\#i... $\rightarrow$...i... & [ri] \\
\hline $\begin{array}{l}\text { engoli } \mathrm{a}_{1} \mathrm{i}_{2} \text { sso } \\
\sigma \sigma^{\prime} \sigma \sigma^{\prime} \sigma \sigma\end{array}$ & 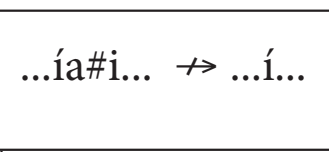 & [liai] \\
\hline $\begin{array}{l}\mathrm{di}_{0} \mathrm{a}_{1} \mathrm{i}_{2} \text { nteiro } \\
{ }^{\prime} \sigma \sigma \tilde{\sigma}^{\prime} \sigma \sigma\end{array}$ & ...ía\#1... $\rightarrow$.. ĩ... & {$\left[\mathrm{d} 3 \tilde{1}^{\tilde{n}^{\mathrm{n}}}\right]$} \\
\hline
\end{tabular}

Quadro 3: Atuação do processo de elisão em relação ao acento de $V_{0}$ e a nasalidade de $V_{2}$

Este comportamento é compatível com observações independentes a respeito do condicionamento da degeminação quando em fronteira de palavras em português. Conforme a análise feita em Ludwig-Gayer (2008, p. 64) sobre o comportamento da degeminação, o acento primário em $V_{1}$ tem um papel neutro, não favorecendo nem desfavorecendo a degeminação (guarani espanhol). Conforme o mesmo trabalho, é o acento em $\mathrm{V}_{2}$ que tende a desfavorecer a degeminação (ele ia). Os exemplos acima mostram isso, pois, em gostaria imensamente, a degeminação se aplicou, apesar de a vogal apagada ser acentuada (cf. BISOL, 2002 a, p. 241, sobre a degeminação em Porto Alegre, e VIANNA, 2009, p.87, sobre a degeminação em Florianópolis). Também no caso de $\mathrm{V}_{2}$ ser nasal, o comportamento é compatível com os resultados observados para o processo de degeminação. Segundo Ludwig-Gayer (2008), estrutura da sílaba de $V_{2}$ não é fator relevante para bloqueio da degeminação. Alguns exemplos do corpus daquele trabalho mostram que a degeminação se aplica sem problemas quando $\mathrm{V}_{2}$ é nasal: coisa que interesse, porque inclusive, noite inteira, aqui em São Borja, a gente incomodava.

Portanto, se a degeminação não é bloqueada nos contextos considerados, reforça-se nossa hipótese de que a realização de seqüências ...ia\#i... como $\rightarrow$...[ii]... seja devida à atuação de fatores de fidelidade, e não de marcação.

Em nosso levantamento de dados, procuramos também por sequências onde $\mathrm{V}_{1}$ fosse precedida por uma vogal posterior alta $\mathrm{e} \mathrm{V}_{2}$ fosse vogal alta posterior, mas nossa amostra só retornou duas ocorrências, sem aplicação de elisão. ${ }^{14}$ Seria interessante termos mais exemplares, entretanto, para isso, teríamos de ter coletas complementares, como as que foram apresentadas para o catalão em Wheeler (2005) (baseado em RECASENS, 1993).

14 As formas são água um e sua, o, nas quais, o contexto se encontra na fronteira entre frases fonológicas, menos favorável à aplicação da elisão. 
Também é pertinente analisar novamente outras amostras estudadas, buscando ocorrências do tipo aqui considerado. Alguns exemplos, retirados do levantamento de Peixoto (2011), da amostra de Lages/SC, do Banco VARSUL, nos quais há contexto, mas não houve aplicação da elisão fri[a\#i] ventando, [ia\#i]studar, [ia\#i]nvadir colôn[ja\#i]taliana -, mostram que, embora ocorrências desse tipo não sejam numerosas em dados de entrevista espontânea, também não podem ser consideradas excepcionais ou de difícil observação.

\section{Ranking e avaliação}

Como vimos, a partir dos exemplos acima, o surgimento de sequências de mesma vogal não bloqueia a aplicação da elisão, o que mostra que o comportamento do processo de elisão é cego ao output. Em teorias orientadas para o output, como a Teoria da Otimalidade (PRINCE e SMOLENSKY, 1993/2004, MCCARTHY e PRINCE, 1995), isto levanta algumas questões, de que vamos tratar aqui. Na próxima seção, mostramos que a não redução de uma sequência /ia\#i/ a [i] implica um tipo de paradoxo de ranking conhecido como chain shift. Para a apresentação desse paradoxo, utilizamos a metodologia de argumentação de ranking proposta em Prince e Brasoveanu (2011). Na seção seguinte, mostramos que esse paradoxo não pode ser resolvido com a abordagem do Serialismo Harmônico (MCCARTHY, 2010a).

\subsection{O paradoxo de ranking}

Como foi demonstrado por Moreton (2003), qualquer alteração entre uma forma de entrada (input) e a forma de saída (output selecionado) tem de funcionar no sentido de aprimorar harmonicamente a forma em questão; caso contrário, essa alteração será descartada pelas restrições de fidelidade. O significado de "aprimoramento harmônico" é que a forma de output tem de ser melhor, menos marcada do que a forma de input em relação a pelo menos uma das restrições de marcação.

Vamos tentar esclarecer essa ideia esquematicamente: consideremos uma restrição de marcação $\mathbf{M}$ que milita contra duas vogais em sequência; se $\mathbf{M}$ domina a restrição de fidelidade correspondente, qualquer entrada com duas vogais em sequência, $V V$, será mapeada em um output $V$. Além disso, uma entrada com três vogais em sequência, VVV, também 
deve ser mapeada em um output de um único V. Se, por outro lado, $\mathbf{F}$ domina $\mathbf{M}$, então, nada deve acontecer, e VVV na entrada deveria ser mapeada para o output VVV.

A nossa observação sobre a resolução de sequências ia\#i apresenta uma espécie de paradoxo de ranking, uma vez que $\mathbf{F}$ parece dominar $\mathbf{M}$ apenas parcialmente, ou vice-versa, $\mathbf{M}$ parece dominar $\mathbf{F}$ apenas parcialmente. Este parece ser um desafio a uma abordagem baseada em restrições, como veremos a seguir. Para que a forma dia inteiro [ii] possa ser gerada, a restrição $\mathrm{M}$ tem de dominar fidelidade ao input, pois, de outra forma, não haveria elisão. Ao mesmo tempo, para que a sequência [ii] seja preservada, paradoxalmente $\mathrm{F}$ tem de dominar $\mathrm{M}$.

Teorias baseadas em regras podem estipular a não-reaplicação de uma regra à sua própria saída. Assim, a não-recursividade de apagamento que exemplificamos acima não chega a ser um problema para esse tipo de abordagem. Entretanto, é preciso estipular o modo não-recursivo de aplicação da regra, já que existem regras que podem reaplicar-se ao seu próprio output (por exemplo, as regras formadoras de estrutura, como as de silabificação, e as regras de aplicação iterativa, como as de harmonia vocálica, por exemplo). Em algumas abordagens, a estipulação do modo de aplicação da regra está na própria definição do tipo de regra. Na teoria de Nespor e Vogel (1986), existe uma distinção entre regras de domínio (span rules) e regras de juntura de domínio. As primeiras aplicam-se somente quando o contexto relevante estiver contido em um domínio determinado pela regra. Nas regras de juntura, o contexto deve ser adjacente a uma juntura entre dois domínios de mesma natureza. No nosso entendimento, a classificação de uma regra como "de juntura" impede a sua reaplicação a uma mesma sequência, pois, depois da primeira aplicação, o contexto deixa de ser rigorosamente falando "de juntura". Seja como for, a abordagem derivacional tem à disposição uma série de recursos que poderiam explicar a aplicação da elisão somente, todos eles apelando para algum tipo de estipulação.

Para a Teoria da Otimalidade, a questão é mais complexa, pois ela não tem como recorrer a dispositivos desse mesmo teor. Formulando o problema de outra forma, observa-se, na língua, que sequências de vogais idênticas, /ii/ ou /i\#i/ realizam-se como [i], pelo processo de degeminação. Isso indica que ${ }^{*} \mathbf{V}_{\alpha} \mathbf{V}_{\alpha}$ domina a restrição de fidelidade relevante, Max ${ }_{V}$ No caso das derivações de diminutivo ( ${ }^{*}$ friinho) e superlativo se observa que a seleção do sufixo também obedece à restrição ${ }^{*} \mathbf{V}_{\alpha} \mathbf{V}_{\alpha}$, ou seja, a restrição que controla a seleção do sufixo tem de estar dominada por ${ }^{\star} \mathbf{V}_{\alpha} \mathbf{V}_{\alpha}$. 
Por outro lado, para que /ia\#i/ emerja como [ii], a restrição de fidelidade deveria dominar ${ }^{*} \mathrm{~V}_{\alpha} \mathrm{V}_{\alpha}$. Para que apenas a elisão se aplique, em gostaria imensamente por exemplo, Onset deveria dominar ${ }^{*} \mathrm{~V}_{\alpha} \mathrm{V}_{\alpha}{ }^{15}$. Além disso, as duas restrições deveriam dominar $\operatorname{Max}_{v}$ caso contrário, nenhum apagamento seria possível. O tableau abaixo está no formato tradicional dos tableaux de violações, porque busca mostrar a seleção do candidato [ii] sobre [iai] com o ranking dado.

\begin{tabular}{|c|c|c|c|}
\hline$/$ ia\#i.../ & Onset & ${ }^{\star} \mathrm{V}_{\alpha} \mathrm{V}_{\alpha}$ & $\operatorname{Max}_{\mathrm{V}}$ \\
\hline a.[ii] & $\star$ & ${ }^{\star}$ & ${ }^{\star}$ \\
\hline b.[iai] & $\star \star$ & & \\
\hline
\end{tabular}

Tableau 3: Tableau de violações mostrando a seleção do candidato [ii]

No entanto, quando consideramos o candidato [i], vemos que ele é selecionado sobre [ii].

\begin{tabular}{|c|c|c|c|}
\hline$/$ ia\#i.../ & Onset & ${ }^{\star} \mathrm{V}_{\alpha} \mathrm{V}_{\alpha}$ & $\operatorname{Max}_{\mathrm{V}}$ \\
\hline a.[ii] & $\star$ & $\star$ & ${ }^{\star}$ \\
\hline b.[iai] & $\star \star$ & & \\
\hline c. [i] & & & $\star \star$ \\
\hline
\end{tabular}

Tableau 4: Tableau de violações mostrando a seleção do candidato [i]

Para evitar que [ii] se torne [i], Max ${ }_{v}$ ou restrição equivalente, deveria dominar ${ }^{*} \mathrm{~V}_{a} \mathrm{~V}_{a}$. Porém, com esse ranking, também não chegaríamos ao candidato pretendido.

Reunimos as informações dos dois tableaux acima em um único tableau, agora no formato comparativo.

\begin{tabular}{|c|c|c|c|}
\hline$/$ ia\#i.../ & Onset & ${ }^{*} \mathrm{~V}_{\alpha} \mathrm{V}_{\alpha}$ & $\mathrm{Max}_{\mathrm{V}}$ \\
\hline a. [ii] $\prec[\mathrm{i}]$ & $\mathrm{L}$ & $\mathrm{L}$ & $\mathrm{W}$ \\
\hline b. [ii] $\prec[$ iai $]$ & $\mathrm{W}$ & & $\mathrm{L}$ \\
\hline
\end{tabular}

Tableau 5: Tableau comparativo

Abaixo, mostramos que, o conjunto de restrições consideradas não consegue selecionar [ii] como output ótimo, independentemente do

15 Em versão apresentada em Collischonn (2011), argumentamos que, na verdade, ${ }^{*} \mathrm{~V}_{\alpha} \mathrm{V}_{\alpha}$ deverá dominar Onset. Esta discussão não será considerada aqui, não sendo crucial para a questão em análise. 
ranking das restrições. A representação utiliza a noção de Condição Elementar de Ranking (ERC, de Elementary Ranking Condition) e de fusão de ERCs (ambas oriundas do trabalho de PRINCE, 2002 e de PRINCE e BRASOVEANU, 2011). Num tableau comparativo, uma ERC corresponde, grosso modo, a uma linha do tableau, e diz quais condições devem ser satisfeitas para que um candidato x sobreviva à comparação com um candidato y. Por exemplo, na linha a. do tableau 6, a ERC é LLW. Ela prediz que, para que [ii] sobreviva à comparação com [i], $\operatorname{Max}_{\mathrm{V}}$ tem de dominar as demais restrições, Onset $e^{\star} \mathrm{V}_{\alpha} \mathrm{V}_{\alpha}$.

\begin{tabular}{|c|c|c|c|}
\hline$/$ ia\#i.../ & Onset & ${ }^{*} \mathrm{~V}_{\alpha} \mathrm{V}_{\alpha}$ & $\mathrm{Max}_{\mathrm{V}}$ \\
\hline a.[ii] $\prec[\mathrm{i}]$ & $\mathrm{L}$ & $\mathrm{L}$ & $\mathrm{W}$ \\
\hline b. [ii] $\prec[$ iai] & $\mathrm{W}$ & & $\mathrm{L}$ \\
\hline Fusão $\mathrm{a}_{\mathrm{b}} \mathrm{b}$ & $\mathrm{L}$ & $\mathrm{L}$ & $\mathrm{L}$ \\
\hline
\end{tabular}

Tableau 6: Tableau comparativo, com fusão de ERCs, mostrando ser impossível a seleção do candidato [ii]

No tableau acima, a última linha representa a fusão das outras duas. Conforme as regras de fusão, quando numa coluna há $\mathrm{L}$ e W, a fusão é a favor do L. ${ }^{16}$ Por isso, o resultado da fusão é uma linha só com Ls, o que mostra que há um paradoxo de ranking, ou seja, não há um ranking possível que selecione o candidato [ii] tanto em relação a [i] quanto a [iai].

A razão por que há esse paradoxo de ranking é a seguinte: a emergência de [ii] a partir de um input /ia\#i/ constitui uma espécie de chain-shift sincrônico. ${ }^{17}$ Trata-se de um tipo de contra-alimentação (ver COLLISCHONN, 2010), relação de opacidade em que uma forma subjacente A se torna $\mathrm{B}$, e uma forma subjacente $\mathrm{B}$ se torna $\mathrm{C}$, mas $\mathrm{A}$ que se torna $\mathrm{B}$ não se torna subsequentemente $\mathrm{C}$ ( $\mathrm{A}, \mathrm{B}$ e $\mathrm{C}$ podem tanto ser segmentos quanto unidades de outro tipo, cf. MORETON E SMOLENSKY, 2002). Na representação abaixo, a linha curva indica o mapeamento inexistente.

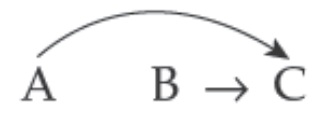

16 Fusão de ERCs é um processo segundo o qual diferentes ERCs são fundidas, de acordo com um conjunto de operações lógicas (BRASOVEANU e PRINCE, 2011). Essa fusão é um elemento importante no Algoritmo de Redução Fusional (FRed) que é uma ferramenta útil na análise da informação contida em um tableau complexo, com múltiplos inputs e seus respectivos candidatos a output, bem como diversas restrições; este algoritmo funciona através da redução da informação redundante; possui aplicações na análise automática de rankings (sendo utilizada nos programas OTSoft e OTHelp, desenvolvidos, respectivamente, por Hayes, Tesar e Zuraw, 2003, e Staubs et al, 2010).

17 Agradeço a Ricardo Bermúdez-Otero a indicação de que se trata de um chain-shift sincrônico. 
Para ilustrar, apresentamos os mapeamentos e, ao lado, as respectivas regras:

\begin{tabular}{|c|c|c|}
\hline a. & iai $\rightarrow$ ii & $a \rightarrow \varnothing / \_\# V \quad$ elisão \\
\hline b. & ii $\rightarrow \mathrm{i}$ & $\mathrm{V}_{\mathrm{i}} \rightarrow \varnothing / \ldots \# \mathrm{~V}_{\mathrm{i}}$ degeminação \\
\hline & iai $\nrightarrow \mathrm{i}$ & \\
\hline
\end{tabular}

O que faz dos processos acima um chain-shift é que ia\#i não passa a i, ou seja, /ia/ não passa, de um só golpe, a $\varnothing$. Lubowicz (2011) classifica casos como esse de "regular chain shift": os mapeamentos A $\rightarrow$ B e B $\rightarrow$ $\mathrm{C}$, que ocorrem ambos independentemente na língua; quando colocados juntos, formariam hipoteticamente uma mudança em cadeia, mas um dos mapeamentos é bloqueado. ${ }^{18}$

A identificação das propriedades de um processo que produz opacidade tem sido considerada importante para o entendimento das razões que estão por trás dos paradoxos de ranking (BAKOVIC, 2007, LUBOWICZ, 2011). Para o entendimento das propriedades de um processo, frequentemente utiliza-se a representação por regras (como fizemos acima) ao lado da representação por restrições; Bakovic (2007) utiliza sistematicamente a representação dos processos através de regras; já Lubowicz (2011) procura representá-los através de restrições esquemáticas. Quando se pensa em regras, diz-se que num processo de chain-shift, uma regra não se aplica, ou seja, há subaplicação. Quando se pensa em restrições, diz se que uma generalização não é, na superfície, verdadeira.

Quando se trata de regras opcionais, poderíamos pensar que o fato de uma regra não se aplicar está relacionado com a sua opcionalidade. $\mathrm{Ou}$ seja, poderíamos pensar que, desde que uma regra seja opcional, por definição, a subaplicação pode acontecer. Com o nosso exemplo, poderíamos pensar que, simplesmente, a regra responsável pelo mapeamento em $\mathbf{b}$ se aplica em algumas formas e em outras não. Nesses casos de subaplicação, é como se a regra não existisse, não fizesse parte da gramática. ${ }^{19}$ Ainda que essa visão pudesse ser considerada para um determinado tipo de opacidade, certamente não é adequada para uma série de processos opacos de

$18 \mathrm{O}$ termo chain shift pode levar a confusão, pois descreve uma não sucessão, uma não-cadeia de mapeamentos, contrariando a expectativa criada pelo nome. Preferimos, por essa razão, manter o termo original em inglês, para indicar o seu caráter de termo especializado.

19 Em outras palavras, modelos de regra variável podem ser pensados como modelos de múltiplas gramáticas: quando a regra se aplica, a gramática inclui a regra; quando a regra não se aplica, a gramática não contém a regra. 
caráter variável (Bakovic, 2011, p.). Além disso, o que estamos buscando mostrar aqui é que a opcionalidade seguramente não é uma forma adequada de abordar a subaplicação em termos de Teoria da Otimalidade, independentemente da abordagem que adotemos para os processos variáveis. ${ }^{20}$ A razão para isso é que um processo não pode meramente deixar de se aplicar; para que isso aconteça, a forma em que o processo subaplica tem de ser selecionada entre as demais formas candidatas em uma gramática determinada. Se não houver ranking que gere essa forma, a subaplicação não pode ser modelada. Portanto, o que vimos defendendo aqui é que as formas com subaplicação, tipo d[ii]nteiro, não podem ser explicadas pela TO Clássica, nem mesmo em uma perspectiva de processo de caráter opcional.

Na próxima seção, vamos considerar a possibilidade de interpretar o problema em questão pelo modelo do Serialismo Harmônico.

\subsection{Serialismo harmônico}

Em trabalhos recentes abordando o tema da opacidade e outros aspectos problemáticos da OT, McCarthy tem defendido o Serialismo Harmônico como solução. Perguntamos se Serialismo Harmônico (MCCARTHY, 2010a), com a sua concepção de etapas sujeitas à exigência de aprimoramento contínuo, poderia ser mais bem equipado para explicar os fatos acima observados. Veremos que não.

No Serialismo Harmônico, a avaliação ocorre em etapas. Não há mudança de ranking entre uma etapa e outra (nesse sentido a proposta é diferente da OT Serial). Em cada etapa, GEN está limitado a fazer apenas uma mudança em relação ao input e a saída de cada passagem por Gen e Eval é submetida como input para uma nova passagem por Gen e Eval. A laçada ${ }^{21}$ Gen $\rightarrow$ Eval $\rightarrow$ Gen ... continua até que não haja mais mudanças possíveis (até que o output ótimo convirja com o input).

Cada etapa na derivação tem de satisfazer a hierarquia de restrições mais satisfatoriamente que a etapa anterior, propriedade denominada aprimoramento harmônico. A hierarquia de restrições é a mesma para todas as iterações (laçadas) Gen/Eval.

20 Pelo menos, dentre as abordagens analisadas em Anttila (2007) e Battisti (2010), nas quais o que varia é o ranking ou o peso dado às restrições dentro de um conjunto delimitado, mas nenhuma restrição é literalmente apagada da gramática.

21 Usamos o termo "laçada" para identificar cada passagem por GEN e Eval, pois o Serialismo Harmônico lembra a sucessividade de etapas iguais dos trabalhos manuais de bordado e crochê. 
No Serialismo Harmônico, uma mudança direta ia\#i >> i não seria possível, pois implicaria duas mudanças simultâneas; a condição é que a mudança seja feita em etapas: primeiro ia\#i $>>$ iai, depois iai $>>$ ii.

O Serialismo Harmônico consegue explicar a realização de ia\#i como [i], pois dá conta das etapas ia\#i $>>$ iai $>>$ ii. No tableau abaixo, usamos uma combinação dos dois tipos de tableau empregados até aqui, o tableau comparativo e o tableau de violações (chamado de "tableau combinado"); as violações estão indicadas com algarismos e não com asteriscos.

Etapa 1

\begin{tabular}{|l|l|l|l|}
\hline$/$ ia\#i.../ & Onset & ${ }^{*} \mathrm{~V}_{\alpha} \mathrm{V}_{\alpha}$ & $\operatorname{Max}_{\mathrm{V}}$ \\
\hline a. $\rightarrow[$ iii $]$ & 1 & 1 & 1 \\
\hline b. [iai] & $1 \mathrm{~W}$ & $\mathrm{~L}$ & $\mathrm{~L}$ \\
\hline
\end{tabular}

Tableau 6: Tableau combinado mostrando a seleção do candidato [ii]

Etapa 2

\begin{tabular}{|l|l|l|l|}
\hline$/$ ii.../ & Onset & ${ }^{*} \mathrm{~V}_{\alpha} \mathrm{V}_{\alpha}$ & $\mathrm{Max}_{\mathrm{V}}$ \\
\hline a. $\rightarrow[\mathrm{i}]$ & & & 1 \\
\hline b. $[\mathrm{ii}]$ & $1 \mathrm{~W}$ & $1 \mathrm{~W}$ & $\mathrm{~L}$ \\
\hline
\end{tabular}

Tableau 7: Tableau combinado mostrando a seleção do candidato [i]

O Serialismo Harmônico, entretanto, não consegue explicar a realização de ia\#i como [ii], que realiza a primeira etapa e não vai adiante. Abaixo, mostramos que, para que isso ocorra, na etapa 2, o output ótimo teria de convergir com o input, devido ao ranking das restrições.

Etapa 1

\begin{tabular}{|l|l|l|l|}
\hline$/$ ia\#i.../ & Onset & ${ }^{*} \mathrm{~V}_{\alpha} \mathrm{V}_{\alpha}$ & $\operatorname{Max}_{\mathrm{V}}$ \\
\hline a. $\rightarrow[$ iii] & 1 & 1 & 1 \\
\hline b. [iai] & $1 \mathrm{~W}$ & $\mathrm{~L}$ & $\mathrm{~L}$ \\
\hline
\end{tabular}

Tableau 8: Tableau combinado mostrando a seleção do candidato [ii]

Etapa 2

\begin{tabular}{|l|l|l|l|}
\hline$/$ ii.../ & Onset & ${ }^{\star} \mathrm{V}_{\alpha} \mathrm{V}_{\alpha}$ & $\operatorname{Max}_{\mathrm{V}}$ \\
\hline a. $\rightarrow[$ ii] $]$ & 1 & 1 & $\mathrm{~W}$ \\
\hline b. $[\mathrm{i}]$ & $\mathrm{L}$ & $\mathrm{L}$ & 1 \\
\hline
\end{tabular}

Tableau 9: Tableau combinado mostrando ser impossível a seleção do candidato [ii] 
No tableau da etapa 2, acima, na ERC correspondente à linha b, podemos ver que há apenas LL e nenhum W, o que, em linguagem comum significa que nenhuma restrição favorece o candidato [ii] sobre [i], ou seja, nenhum ranking permitiria que o candidato [ii] prevalecesse sobre o candidato [i].

Portanto, com as restrições que estamos usando, a proposta do Serialismo Harmônico, com etapas de derivação sucessiva, não consegue melhores resultados do que a OT Clássica, no que se refere a este aspecto do sândi aqui examinado. Ambas as propostas predizem que uma mudança / ia\#i/ $\rightarrow$ ii não seja possível, contrariando o que nos dizem os dados.

O problema parece estar no conjunto de restrições que estamos usando. Se tivermos a restrição correta, o mapeamento a ii $\rightarrow$ [i] pode ser bloqueado na etapa 2 e, assim, ou output pretendido [ii] será alcançado. Porém, a solução não é tão simples assim: a restrição proposta não deve impedir o mapeamento /ia\#i/ $\rightarrow$ ii na etapa 1 .

McCarthy (2010a) reconhece que os casos de chain shift sincrônico são problemáticos para o Serialismo Harmônico. Uma das soluções sugeridas pelo autor seria adotar a proposta de McCarthy (2007) para o tratamento da opacidade, com restrições de PRECEDÊNCIA. Nesta proposta, uma variante do Serialismo Harmônico, há restrições que determinam a ordem em que duas restrições de fidelidade serão violadas em uma cadeia de candidatos. Por exemplo, uma restrição de PRECEDÊNCIA poderia dizer que uma violação à restrição de fidelidade hipotética $\mathrm{Max}_{\mathrm{i}}$ tem de preceder em termos de derivação a violação à restrição de fidelidade a $\mathrm{Max}_{\mathrm{a}}$. Assim, [i] não poderia ser apagado depois do apagamento de /a/. Mas essa solução implicaria a necessidade de adotar restrições do tipo PRECEDÊNCIA para fenômenos variáveis, uma ideia que não parece coadunar-se com a teoria proposta em McCarthy (2007).

Outra solução sugerida pelo autor é determinar se, em casos de chain-shift não ocorre, na realidade, uma neutralização aparente. Em termos do fenômeno que estamos analisando, significaria perguntarmos se a sequência de duas vogais [ii] que resulta de apagamento aplicado a ia\#i é idêntica a sequências [ii] que estão sujeitas a apagamento. Uma observação desse tipo não foi feita até o momento e extrapolaria os propósitos deste trabalho. Embora essa linha de abordagem pudesse ser possível no caso que estamos analisando, não nos parece que, via de regra, seja uma solução para casos de chain shift.

\section{Conclusões e projeções}

O foco principal desta análise foi o comportamento de sequências em que $\mathrm{V}_{1}$ é precedida por uma vogal anterior alta, como no exemplo "dia in- 
teiro". Neste trabalho, a argumentação baseia-se no ponto de vista de que o processo de elisão é um efeito de restrições em conflito. Dessa perspectiva, em sequências do tipo /...ia\#i.../, esperar-se-ia que a elisão não se aplicasse, pois o resultado, ou seja [...ii...], é uma sequência proibida ou pouco tolerada no português brasileiro, e espera-se a atuação de restrições contrárias a sequências desse tipo.

Entre as evidências que atestam a má-formação de sequências [ii] estão processos de degeminação em fronteira de palavra e também bloqueios de formação morfológica. Esperar-se-ia, portanto, que as restrições responsáveis por degeminação e bloqueio morfológico também atuassem no sentido de evitar a aplicação da elisão sempre que o resultado fosse uma sequência de vogais idênticas. Contrariamente às expectativas, os dados mostram que a elisão acontece nesses contextos (d[ii]nteiro). ${ }^{22}$

Apresentamos o modo como as restrições dão conta do processo de elisão em uma análise otimalista clássica e mostramos que as sequências ia\#[i] levantam o problema do paradoxo de ranking. Na sequência, consideramos a proposta alternativa de McCarthy (2010a), o Serialismo Harmônico, e constatamos que também resulta em problema de ranking, pois com as restrições previstas, o output sempre levaria a uma redução total da sequência /ia\#i/ a [i].$^{23}$ Constatamos que a razão por que a proposta não funciona é o fato de se tratar de um tipo de chain shift, fenômeno que até o momento não recebeu uma análise satisfatória dentro do Serialismo Harmônico. A discussão aponta para análises suplementares e indica caminhos para uma possível solução do impasse em abordagens da OT.

\section{BIBLIOGRAFIA}

ANTTILA, Arto. Variation and optionality. In: DE LACY, Paul (ed.), The Cambridge Handbook of Phonology, Cambridge University Press, Cambridge, 2007. p. 519-536.

BAKOVIC, Eric. A revised typology of opaque generalizations. Phonology 24 (2007). p. 217-259.

BATTISTI, Elisa . Variação. In: BISOL, Leda; SCHWINDT, Luiz Carlos da Silva. (Org.). Teoria da Otimidade: Fonologia. 1ed.Campinas: Pontes Editores, 2010, v. 1, p. 271-290.

BISOL, Leda. A degeminação e a elisão no VARSUL. In: BISOL, L.;

22 É possível acontecer também a degeminação (d[i]nteiro), mas o que buscamos mostrar aqui é que a estrutura [ii] não é evitada.

23 Como se trata de processo variável, a outra alternativa seria a não aplicação de elisão; no entanto a forma com elisão e manutenção das duas vogais não poderia ser produzida. 
BRESCANCINI, C. (orgs.) Fonologia e variação: recortes do português brasileiro. Porto Alegre: EDIPUCRS, 2002. p. 231-250.

BISOL, Leda. Sândi externo: o processo e a variação. In: KATO, M. (org.) Gramática do português falado. Volume V: Convergências. $2^{\mathrm{a}}$ ed. Campinas, SP: Editora da UNICAMP, 2002b. p. 53-97.

BISOL, Leda. Sandhi in Brazilian Portuguese. Probus (Dordrecht), Mouton de Gruyter, Berlin, v. 15, n. 2, p. 177-200. 2003

BRASOVEANU, A., and PRINCE, Alan. Ranking and Necessity: the Fusional Reduction Algorithm. Natural Language and Linguistic Theory, v. 29, n.1, 2011. p. 3-70.

CASALI, Roderic. F. Vowel Elision in Hiatus Contexts: Which Vowel Goes? Language, 1997. p. 493-533

COLLISCHONN, G. Sândi vocálico no português brasileiro: uma aplicação para o Serialismo Harmônico (McCarthy, 2008)? In: LEE, S.H. (org.). Vogais além de Belo Horizonte. Belo Horizonte: FALE/UFMG, 2012. p. $42-56$ [online] disponível em: http://http://www.letras.ufmg.br/site/elivros.asp COLLISCHONN, Gisela. A Elisão em Hiato no Português Brasileiro e o Tratamento das Sequências Vocálicas Resultantes. Trabalho apresentado no XVI Congreso Internacional de la ALFAL, 2011, Alcalá de Henares. HAYES, Bruce; TESAR, Bruce; ZURAW, Kie. OTSoft 2.3.1. [online] disponível em http://www.linguistics.ucla.edu/people/hayes/otsoft/ KIPARSKY, Paul Variable rules. Handout distribuído no Rutgers Optimality Workshop 1, 1993. [online] disponível em http://www.stanford. edu/ kiparsky/Papers/nwave94.pdf

$\mathrm{KROCH}$, Anthony. Reflexes of grammar in patterns of language change. Language Variation and Change, 1, 1989. p.:199-244. LUBOWICZ, Anna. Chain Shifts. In: Marc van Oostendorp et al., ed., The Blackwell Companion to Phonology. Wiley-Blackwell, 2011. p. LUDWIG-GAYER, Juliana. Os processos de sândi externo: análise variacionista da fala de São Borja. 2008. Dissertação (Mestrado em Letras), Universidade Federal do Rio Grande do Sul, 2008. [online] disponível em: http://www.lume.ufrgs.br/handle/10183/14993.

MCCARTHY, John J. Hidden Generalizations. London: Equinox Publishing, 2007.

MCCARTHY, John. J. Harmonic Serialism Supplement to Doing Optimality Theory. The Selected Works of John J. McCarthy. 2010a. [online] disponível em http://works.bepress.com/john_j_mccarthy/108 MCCARTHY, John. J. Studying GEN. Journal of the Phonetic Society of Japan, v.13, n.2, p.3-12, 2010 b. 
MCCARTHY, John. J. Perceptually Grounded Faithfulness in Harmonic Serialism. Linguistic Inquiry, Vol. 42, No. 1 . p. 171-183, 2011.

MCCARTHY, John. J. Doing Optimality Theory. Applying Theory to Data. Oxford, Malden/MA, Carlton: Blackwell, 2008a.

MCCARTHY, John. J. The serial interaction of stress and syncope. Natural Language \& Linguistic Theory, 26, p 499-546, 2008b.

MORETON, Elliot. Non-computable functions in Optimality Theory. In: MCCARTHY, J.J. (ed.), Optimality Theory in Phonology. A Reader. Oxford: Blackwell, 2003.p. 141-164. NOGUEIRA, Milca. V. Aspectos Segmentais dos Processos de Sândi Vocálico Externo no Falar de São Paulo. 2007. Dissertação (Mestrado em Letras) Universidade de São Paulo, São Paulo, 2007.

PAOLILLO, John C. Analyzing Linguistic Variation. Statistical Models and Methods. Stanford, California: Center for the Study of Language and Information - Lecture Notes, 2001. PEIXOTO, Bruna L. Processos vocálicos no PB: elisão no Português falado em Lages - SC. Comunicação no XXIII Salão de Iniciação Científica, Porto Alegre: UFRGS, 2011.

PRINCE, Alan. Arguing Optimality. In: CARPENTER, A; COETZEE, A.; DE LACY, P. (eds.) University of Massachusetts Occasional Papers in Linguistics 26: Papers in Optimality Theory II. Amherst, Massachusetts: GLSA Publications, 2002. p. 269-304. [online] disponível em Rutgers Optimality Archive, roa.rutgers.edu.

QUEDNAU, L.R. Os ditongos do latim ao português. Letras de Hoje, v. 40, no 3, setembro 2005, p.89-99.

STAUBS, Robert; BECKER, Michael; POTTS, Christopher; PRATT, Patrick; McCarthy, John J.; PATER, Joe. OT-Help 2.0. Software package. Amherst, MA: University of Massachusetts Amherst. 2010. [online] disponível em web.linguist.umass.edu/ OTHelp/

TAGLIAMONTE, Sali A. Analysing Sociolinguistic Variation. Cambridge: Cambridge University Press, 2006.

TENANI, Luciani. E. Domínios prosódicos no Português. 2002.Tese (Doutorado em Letras), Universidade Estadual de Campinas, IEL, Campinas, 2002. VIANNA, Paula. Sândi vocálico externo : o processo e a variação na cidade de Florianópolis-SC. 2009. Dissertação (Mestrado em Letras), Universidade Federal do Rio Grande do Sul, Porto Alegre, 2009. [online] disponível em: http://www.lume.ufrgs.br/handle/10183/18353

VIGÁRIO, Marina. The Prosodic Word in European Portuguese, Berlin \& New York: Mouton de Gruyter, 2003. 
WHEELER, Max. The Phonology of Catalan. Oxford: Oxford University Press, 2005.

Recebido em: 22/03/2013; Aceito em: 18/06/2013

\section{Nota:}

1 Sob o rótulo de "peso relativo", apresentamos o valor de input, retirado da rodada de análise pelo programa VARBRUL/GoldVarb. 